\title{
Chikungunya Fever and Its Impact: Bangladesh Perspective
}

\author{
Sazzad Bin Shahid \\ Associate Professor, Department of Microbiology, Dhaka Medical College, Dhaka, Bangladesh; Email: sazzadshahid@gmai.com; \\ Cell no.: +88017148384165
}

Bangladesh is a densely populated developing country. There are still a good number of communicable diseases prevalent in the community. Among these some of the diseases create a disease burden in the society. Chikungunya Fever is one of these.

Chikungunya Fever is a viral infection and it is caused by the RNA virus named chikungunya virus. It is an arbovirus (Arthropod Born Virus). This virus is transmitted by Aedes mosquito. It is very interesting that Aedes mosquito is more prevalent in Bangladesh. There are many safe breeding places for this mosquito. Therefore, this virus can easily infect at any age group of the community. In these context development guidelines is an important issue.

Entomological Examination has proved that Aedes aegypti is the common Vector responsible for transmission in urban areas whereas Aedes albopictus has been implicated in rural areas. It has been established that the Aedes mosquito breeds in domestic setting such as flower vases with clean water, water storage containers air coolers water reservoir, construction sites, coconut shells, containing clean water, tures, plastic, metal cans which is stored more than three days.

In the South-East Asia region, chikungunya virus persists in the human population by human mosquito human transmission cycle. During post monsoon season a high vector density leads to increase the transmission Communicable Disease control (CDC) of Directional General of Health services under Ministry of Health \& Family welfare of Bangladesh with the collaboration of IEDCR has planned to develop the management guidelines for chikungunya fever. These guidelines have given the proper diagnosis \& treatment. In Bangladesh the chikungunya a virus is confirmed by RT-PCR. Furthermore, the ICT devices are also available to detect the chikungunya virus specific antibody which is both IgG and IgM.

There is a dilemma of clinical diagnosis of chikungunya fever. The reason is that clinical features of chikungunya fever has the similarities with Dengue fever leads to diagnostic confusion and management of the causes. The main cardinal features of chikungunya fevers high fever, rash, body ache with arthritis or joint manifestation, vomiting is also an important symptom of chikungunya fever. This leads to Electrolyte imbalance. However, bleeding manifestation is one of the important complication of Dengue fever which is absent in chikungunya fever is only symptomatic treatment. There is no current specific treatment available for chikungunya fever. Like Dengue fever, fluid management is essential tools for primary management with application of Paracetamol. This drug gives the relief of pain as well as high fever. All these management is maintained in the guidelines. Thus this guideline will give a management tools to the primary to tertiary care hospital in Bangladesh.

[Bangladesh Journal of Infectious Diseases, December 2019;6(2):28]

Cite this article as: Shahid SB. Chikungunya Fever and Its Impact: Bangladesh Perspective. Bangladesh $\mathbf{J}$ Infect Dis 2019;6(2):28 